\title{
Ideação Suicida, Resolução de Problemas, Expressão de Raiva e Impulsividade em Dependentes de Substâncias Psicoativas
}

\author{
Suicidal Ideation, Problem Solving, Expression of Anger and Impulsiveness \\ in Dependents on Psychoactive Substances
}

\author{
Rosa Maria Martins de Almeida*, $a$, Antoniéle Carla Stephanus Flores ${ }^{b} \&$ Morgana Scheffer $^{b}$ \\ ${ }^{a}$ Universidade Federal do Rio Grande do Sul, Porto Alegre, Brasil \\ $\&{ }^{b}$ Universidade do Vale do Rio dos Sinos, São Leopoldo, Brasil
}

\begin{abstract}
Resumo
Este estudo teve por objetivo comparar homens dependentes de substâncias psicoativas, com não dependentes, quanto às funções executivas e à expressão emocional e comportamental relacionando com a presença de ideação suicida. A amostra foi composta por 25 dependentes de substâncias psicoativas e 25 não dependentes. Empregou-se na coleta de dados um questionário sociodemográfico e de aspectos de saúde, a Entrevista Diagnóstica (MINI-Plus), o Inventário de Expressão de Raiva como Estado e Traço, a Escala de Impulsividade de Barratt (BIS-11), o Teste Wisconsin de Classificação de Cartas e a Escala de Ideação Suicida de Beck. Conclui-se que os dependentes de substâncias psicoativas do estudo não apresentaram alterações cognitivas significativas, o que não vai ao encontro da literatura, porém apresentaram alterações quanto à impulsividade e à expressão de raiva.

Palavras-chave: Suicídio, drogas, expressão de raiva, impulsividade, raciocínio abstrato.
\end{abstract}

\begin{abstract}
This study aimed to compare men with substance use disorders and those who are no addicted/non-dependent on drugs in terms of their executive functions, emotional and behavioral expressions giving particular emphasis on the presence of suicidal ideation. The sample consisted of 25 individuals with substance use disorders and 25 non-dependent individuals. The data collection consisted of a questionnaire including sociodemographic and health data, the Diagnostic Interview (MINI-Plus), the Anger Expression Inventory - State and Trait (STAXI), the Barratt Impulsiveness Scale (I-BIS11), the Wisconsin Card Sorting Test (WCST) and the Beck Scale for Suicide Ideation (BSI). The data revealed that the substance dependent individuals showed no significant cognitive impairment, fact that does not math with the literature. However, they presented alterations with respect to impulsivity and expression of anger.

Keywords: Suicide, drugs, expression of anger, impulsivity, abstract reasoning.
\end{abstract}

O suicídio é uma das maiores causas de mortalidade no mundo, especialmente, entre os jovens (McGirr, Renaud, et al., 2007). Com sua crescente prevalência, esta condição tem sido considerada uma questão de saúde pública (Hawton \& Heeringen, 2002). Um estudo epidemiológico de prevalência descreveu variações interculturais e por faixa etária, no qual registrou que, de modo geral, a prevalência de mortes por suicídio é mais elevada nos países da Europa Oriental, mas baixa em países das Américas Central e do Sul. Tradicionalmente, a taxa de mortalidade por suicídio sempre foi maior entre adultos. Entretanto, nas últimas décadas no Brasil, evidencia-se um aumento de casos em faixas etárias mais jovens, ocupando na atua lidade o terceiro lugar entre as causas de mortalidade em

* Endereço para correspondência: Universidade Federal do Rio Grande do Sul, Porto Alegre, Brasil. E-mail: rosa_almeida@yhaoo.com indivíduos entre 15 e 34 anos de ambos os sexos (Almeida, Guedes, Nogueira, França, \& Silva, 2009).

Além do crescente aumento de mortes por suicídio, as tentativas de suicídio são duas vezes mais prevalentes no sexo feminino, quando comparadas aos homens (Romão \& Vieira, 2004). O problema central é que a maioria dos casos de violência reincidirá, com igual ou superior gravidade, se não houver alguma ação que interrompa sua dinâmica. Resultados do National Comorbidity Survey (NCS) indicam que cerca de $5 \%$ da população geral norte-americana tentou o suicídio em algum momento da vida. De modo similar, tentativas de suicídio estão associadas a significativas morbidades e constituem um preditor maior de suicídio posterior (Diaconu \& Turecki, 2008; World Health Organization [WHO], 2007).

Seis fatores de risco foram descritos como as mais importantes no comportamento suicida: o aumento na prevalência de transtornos depressivos; o aumento do uso 
abusivo de substâncias psicoativas; mudanças psicobiológicas, como a diminuição na data de início da puberdade; aumento no número de estressores sociais; mudança nos padrões de aceitação de comportamentos suicidas e aumento das taxas de tentativas de suicídio (Diekstra \& Garnefski, 1995; Rogers, 2001; Weir, 2001).

Transtornos psiquiátricos e drogadição estão presentes em 90\% dos suicídios nos países como Europa e Estados Unidos (Moscicki, 1995). Dados de pesquisa relacionada à associação entre uso de álcool e suicídio mostram que as taxas de suicídio estão inversamente relacionadas com o grau de interação social e que o uso abusivo de álcool tem como consequência a desaprovação social e gradativa deterioração dos laços sociais (Meneghel, Victora, Faria, Lenine, \& Falk, 2004).

Algumas linhas de evidência sugerem que a maioria dos sujeitos que comete suicídio apresenta comportamentos impulsivos e impulsivo-agressivos (Mann, Oquendo, Underwood, \& Arango, 1999; Mann, Waternaux, Haas, $\&$ Malone, 1995). Isto é consistente com alguns estudos que vêm indicando haver, em várias categorias diagnósticas, uma correlação positiva entre a presença de traços impulsivos ou impulsivo-agressivos e a tendência a tentar manifestar o comportamento suicida (Corruble, Damy, \& Guelfi, 1999; Malone, Haas, Sweeney, \& Mann, 1995).

Estudos têm encontrado um aumento no córtex préfrontal do binding de agonistas serotoninérgicos a receptores pós-sinápticos de serotonina, principalmente, o receptor 2A (Arango et al., 1990; Hrdina, Demeter, Vu, Sotonyi, \& Palkovits, 1993). Isto sugere uma up regulation compensatória em resposta a uma atividade serotoninérgica reduzida nesta região cerebral, o que é de grande interesse já que o córtex pré-frontal está envolvido na execução da função inibidora. Lesões nesta área resultam em desinibição do impulso e do comportamento agressivo (Mann, 1998), portanto, é possível que sujeitos com uma redução serotoninérgica nesta região cerebral possam ter uma maior predisposição a comportamentos impulsivos e de auto-agressão, quando expostos a situações estressantes, tais como: quadros psiquiátricos e, em algumas instâncias, isto poderia resultar na manifestação de comportamento suicida (Turecki, 1999). Estudo realizado nos Estados Unidos com prisioneiros verificou que indivíduos que tentaram o suicídio apresentaram acusações de crimes violentos e relação com drogas e intoxicação de álcool (Felthous, 2011).

Comportamentos agressivos e impulsivos estão presentes não só em indivíduos com depressão maior e suicídio (Malone et al., 1995; Pompili et al., 2008), mas também em vitimas de suicídio que apresentavam outros diagnósticos, tais como Abuso de Substâncias (Brent, 1993; Brent, Bridge, Johnson, \& Connolly, 1996; Dumais et al., 2005) e Transtorno de Personalidade Borderline (McGirr, Paris, Lesage, Renaud, \& Turecki, 2007; McGirr, Renaud, Seguin, Alda, \& Turecki, 2008). Estudo realizado por Reyes et al. (2011) mostrou que indivíduos poliusuários de drogas apresentam maior propensão ao suicídio, as- sim como, o uso de álcool isoladamente contribui para tal comportamento. Em relação a drogas ilícitas, homens com ideação suicida parecem ser mais suscetíveis do que homens sem ideação suicida para satisfazer critérios de uso de cocaína (Cottler, Campbell, Krishna, CinninghamWilliams, \& Abdallah, 2005). Corroborando com estudo acima citado, Tiet, Ilgen, Byrnes, e Moos (2006) buscaram identificar o perfil do paciente usuário de droga que apresentava elevado risco de suicídio. Os resultados mostraram que os fatores incluídos para o risco de suicídio nessa população foram: história prévia de tentativa de suicídio, consumo de cocaína, ideação suicida e dificuldade em controlar comportamentos violentos.

Uma das áreas cerebrais que tem sido repetidamente implicada na conduta violenta é o córtex frontal (Filley, Price, Nell, Antoinette, \& Morgan, 2001). Especificamente, a área pré-frontal modula as atividades do hipotálamo e do sistema límbico e seu funcionamento tem um papel crítico na organização do comportamento, na linguagem, nas ações cognitivas e na tomada de decisão (Fuster, 2002). Estas funções cognitivas implicam no desempenho do lobo pré-frontal em termos de controle antecipado da ação, da escolha dos objetivos a serem alcançados, do planejamento, da tomada de decisão, da seleção da resposta mais adequada e da inibição de outras, da atenção no acompanhamento enquanto a ação ocorre e da verificação do resultado (Gil, 2002). Prejuízos neuropsicológicos e do comportamento podem predispor pacientes a praticarem atos autoprejudiciais (Raust et al., 2007). Dessa forma, a prevenção desse comportamento autolesivo necessita focar-se na maior regulação da influência das alterações emocionais e das estratégias de resolução de problemas (Ohmann et al., 2008).

Dessa forma, o presente estudo teve como objetivo comparar homens dependentes de substâncias psicoativas, com não dependentes, em relação a funções executivas, alterações emocionais e do comportamento como: expressão de raiva, impulsividade, raciocínio abstrato e a capacidade para gerar estratégias de solução de problemas, relacionando com a presença de ideação suicida.

\section{Método}

\section{Delineamento}

Trata-se de um estudo transversal, ex-pos-facto, descritivo, correlacional, com análise quantitativa dos dados.

\section{Participantes}

A amostra foi constituída por 50 homens de 18 a 40 anos de idade, provenientes da Grande Porto Alegre, sendo 25 homens dependentes de substâncias psicoativas com idade média de 27,2 $(D P=7,21)$, em abstinência por duas semanas a dois meses $(M=48,52 ; D P=12,98)$, provenientes de um Centro de Triagem para dependentes químicos, e 25 homens, com idade média de 26,4 $(D P=7,12)$ não dependentes de substâncias psicoativas que foram pareados por idade e classe econômica. Foi realizado 
cálculo amostral que inferiu o número de 21 participantes. Porém, foi possível obter participação maior, totalizando 25 entrevistados, correspondendo a $50 \%$ da população total da instituição sem uso de medicação. O cálculo do tamanho da amostra foi feito utilizando-se o programa Statcalc do Epi-Info versão 3.4 para um nível de confiança de $95 \%$.

Foram excluídas da amostra, pessoas com diagnóstico de deficiência mental, com diagnóstico de esquizofrenia, previamente avaliados através da entrevista diagnóstica Mini International Neuropsychiatric Interview (MINIPlus; Amorim, 2000). Os não alfabetizados, com problemas visuais ou auditivos e em uso de medicação (ansiolíticos e antidepressivos). Além desses critérios de exclusão, os sujeitos controles não podiam fazer uso de cigarro.

\section{Instrumentos}

Questionário Sociodemográfico, contendo questões que investigaram os aspectos sociodemográficos e de saúde, tais como uso de medicamentos, história clínica pregressa, problemas de audição e visão diagnosticados, dos participantes da pesquisa.

Escala de Ideação Suicida de Beck (BSI; adaptação e normatização brasileira: Cunha, 2001), considerada uma escala de auto-avaliação usada para detectar a intensidade da ideação suicida nos pacientes baseada na última semana, sendo que foi utilizada a versão validada em português no Brasil. As propriedades psicométricas dessa escala, na versão em português, são consideradas satisfatórias tanto em amostras clínicas quanto em amostras não clínicas. Em termos de validade, os coeficientes alfa para o instrumento, variam desde 0,59 a 0,62.

Inventário de Expressão de Raiva como Estado e Traço (STAXI; adaptação e normatização brasileira: Biaggio, 1992/2003) Este instrumento avalia a agressividade através da mensuração de experiências e expressões de raiva. A escala é composta por 44 itens e o formulário de aplicação possui três subescalas, todas apresentam coeficientes alfa maiores que 0,60 .

Escala de Impulsividade de Barratt (Diemen, Szobot, Kessler, \& Pechansky, 2007), é um dos métodos mais utilizados para medir a impulsividade, porém esta escala não foi validada para o Português, o que gera dificuldades para a realização de estudos neste contexto. O BPI é uma escala de auto-aplicação, composta por 30 itens do tipo Likert, com perguntas que fornecem uma pontuação total de impulsividade e três sub-pontuações: atenção, falta de planejamento por impulsividade e de impulsividade motora. A pontuação varia de 30 a 120 e não existe ponto de corte estabelecido. Um estudo realizado em 2007 teve como objetivo adaptar para o português e realizar a validação de construto da Escala de Impulsividade de Barratt para adolescentes. O coeficiente de correlação intraclasse foi de 0,90 e a consistência interna foi de 0,62 para os 30 itens.
Teste Wisconsin de Classificação de Cartas (WCST; adaptação e normatização brasileira: Cunha et al., 2005), criado em 1948, ampliado e revisado posteriormente. Foi utilizado para avaliar o raciocínio abstrato e a capacidade do sujeito para gerar estratégias de solução de problemas, em resposta a condições de estimulação mutáveis. Também pode ser considerada uma medida da flexibilidade do pensamento. Os coeficientes alfa variam de 0,39 a 0,72, com média de 0,57 e com uma mediana de 0,60 .

Mini International Neuropsychiatric Interview (MINIPlus; adaptação e tradução: Amorim, 2000), foi utilizado como instrumento de triagem e consiste em uma entrevista diagnóstica padronizada breve, compatível com os critérios do DSM-III-R/IV e da CID-10. A versão Plus do MINI, mais detalhada, gera diagnósticos positivos dos principais transtornos psicóticos e do humor do DSMIV. Questionamentos sobre o uso de substâncias psicoativas estavam direcionados aos últimos 12 meses. Estudos de confiabilidade e validade foram desenvolvidos, comparando o MINI ao SCID-P e ao CIDI. Os resultados desses estudos mostram que o MINI apresenta índices de confiabilidade e de validade comparáveis aos dos instrumentos referidos.

\section{Procedimento}

A avaliação ocorreu de forma individual, pela autora do estudo capacitada para tal, primeiramente, junto a um centro de triagem para dependentes químicos, localizado na grande Porto Alegre (RS), sendo a ordem dos testes aplicados de forma aleatória, a fim de evitar viés relacionado à ordem de aplicação. Os instrumentos foram aplicados no centro de triagem para tratamento da dependência de substâncias psicoativas, durante a permanência dos pacientes no mesmo em uma sessão única de aproximadamente uma hora e meia. Na segunda parte do presente estudo, referente ao grupo controle, a pesquisadora entrou em contato e convidou os funcionários de uma prestadora de serviços terceirizados, também localizada na grande Porto Alegre (RS). Foram controlados a faixa etária, a classe econômica e o não uso de substâncias psicoativas. Este critério foi constatado no primeiro contato com o possível candidato ao grupo controle. Caso não fosse constatado o pareamento, a aplicação dos instrumentos não ocorria.

Não houve recusa dos controles e estes responderam os instrumentos, em uma sessão única, também com duração de aproximadamente uma hora e meia, sendo a ordem dos testes aleatória. $\mathrm{O}$ ambiente de aplicação era reservado, com iluminação apropriada e sem a presença de ruídos. O projeto foi aprovado pelo Comitê de Ética da Universidade do Vale do Rio dos Sinos (resolução 041/ 2009), sendo adotados todos os procedimentos éticos para a realização da pesquisa com seres humanos (risco mínimo, direito de sigilo, consentimento informado), de acordo com a American Psychological Association (APA), respeitando a dignidade e o direito das pessoas. A aplica- 
ção dos instrumentos ocorreu após apresentação do Termo de Consentimento Livre e Esclarecido ao participante da pesquisa e sua assinatura, concordando com a participação na pesquisa.

\section{Análise de Dados}

Os dados foram tabulados em planilha eletrônica do banco do Software estatístico SPSS (Statistical Package for Social Sciences) versão 16.0 e analisados. Primeiramente, os dados foram submetidos a procedimentos de estatística descritiva. Para comparação entre os dois grupos, foi utilizado o teste $t$ de Student e Qui-Quadrado (para dados de frequência) e para as correlações utilizouse coeficiente de correlação de Spearman. O nível de significância aceito foi de $p \leq 0,05$.

\section{Resultados}

Nenhum participante estava utilizando medicações no momento da aplicação dos instrumentos e não houve relato de doenças psiquiátricas, através do questionário sócio-demográfico e dos aspectos de saúde. No grupo controle $(n=25)$, a média de idade dos participantes foi de 26,4 anos $(D P=7,12)$ e, no grupo dos dependentes de substâncias psicoativas $(n=25)$, a média de idade dos participantes foi de 27,2 anos $(D P=7,21)$. A classe econômica predominante nos dois grupos foi " $\mathrm{C}$ ", que corresponde à classe baixa (48\%), segundo dados do questionário sociodemografico aplicado. Com relação à escolaridade, o grupo dos dependentes possuía nível fundamental incompleto (28\%) e nível médio incompleto $(28 \%)$ e o grupo controle nível médio completo $(60 \%)$.

No grupo dos dependentes de substâncias psicoativas é relevante apontar a idade do primeiro uso de drogas $(M$ $=13,24 ; D P=2,96$ ), e o tempo de abstinência no momento da entrevista, que foi, em média, de 48,52 dias $(D P=12,98)$. As drogas mais utilizadas por eles foram cigarro (92\%), cocaína (92\%), maconha $(88 \%)$, álcool $(88 \%)$ e crack $(88 \%)$, seguidas por cola $(28 \%)$, heroína (4\%), LSD (4\%) e ecstasy (4\%). Entre os dependentes, $44 \%, 56 \%$ e $60 \%$ usavam maconha, cigarro e álcool há nove anos ou mais respectivamente. O crack e a cocaína foram utilizados por $56 \%$ e $32 \%$ dos dependentes, respectivamente nos últimos cinco anos. Trinta e dois por cento relataram que consumiam maconha 4 vezes/mês e $40 \%$ relataram que o consumo de álcool era de duas a seis vezes por semana e $28 \%$ utilizavam cocaína e também o crack todos os dias, várias vezes ao dia. Apenas $8 \%$ dos dependentes apresentavam HIV. Em ambos os grupos não foram detectados critérios diagnósticos para transtornos psiquiátricos.

Em relação à ideação suicida, dos 25 homens dependentes de substâncias psicoativas entrevistados, 24\% apresentaram ideação e $76 \%$ não apresentaram. O mesmo aconteceu com as tentativas de suicídio onde $24 \%$ dos dependentes entrevistados apresentaram tentativas e $76 \%$ não apresentaram. Nenhum participante do gru- po controle apresentou ideação suicida ou tentativa de suicídio. Na comparação da freqüência entre o grupo controle e o grupo de dependentes, pode-se observar que o grupo com maior prevalência e estatisticamente significativa para a variável ideação suicida e tentativa de suicídio, segundo resultados da BSI, foi o grupo de dependentes, com $(p<0,001)$.

Em relação à variável expressão de raiva, avaliada através do STAXI, na comparação entre os grupos, o grupo de dependentes apresentou diferença estatisticamente significativa na comparação com o grupo controle, ou seja, maior traço de raiva, temperamento de raiva, reação de raiva,e expressão de raiva e menor controle da raiva. Pode-se também observar, que na comparação das médias entre os dois grupos, em relação à variável impulsividade, avaliada através da Escala de Impulsividade de Barrat, o grupo de dependentes apresentou resultados estatisticamente significativos nas sub-variáveis: atenção, impulsividade motora, falta de planejamento por impulsividade e escore total de impulsividade. A capacidade para gerar estratégias de solução de problemas foi avaliada através do teste Wisconsin, que teve como resultados estatisticamente significativos para o grupo de dependentes, os erros não perseverativos e para o grupo controle, a resposta de nível conceitual. Na Tabela 1 estão expostos os dados de comparação entre os grupos e os instrumentos aplicados.

A Tabela 2 mostra os valores das correlações entre as variáveis avaliadas neste estudo, que foram: ideação suicida correlacionada com expressão de raiva, impulsividade e estratégias de solução de problemas. A correlação com a variável ideação suicida e capacidade para gerar estratégias de solução de problemas não apresentou itens significativos, com exceção do número de ensaios administrados. Da mesma forma, correlação significativa foi encontrada entre ideação suicida e tempo de uso do crack $(r=0,500 ; p=0,011)$.

\section{Discussão}

Os resultados mostraram que homens dependentes de substâncias psicoativas apresentaram ideação suicida em $24 \%$ da amostra estudada, em comparação ao grupo controle, que não apresentou ideação suicida. Esses resultados corroboram achados de outros estudos sobre a relação entre abuso de substâncias e ideação suicida, os quais mostraram que o uso do álcool ou outras substâncias de abuso são associados com maiores níveis de ideação suicida (Grant \& Hasin, 1999; Knesper, 2003; Lecrubier, 2001; Pages, Russo, Roy-Byrne, Ries, \& Cowley, 1997).

Porém, na maioria das pesquisas revisadas, a porcentagem de indivíduos dependentes apresentando ideação suicida é maior daquela encontrada neste estudo. Pode ser postulado que esse número seja decorrente do pequeno número da amostra estudada e de sua heterogeneidade, não sendo possível a generalização dos dados. Entretan- 
Almeida, R. M. M., Flores, A. C. S. \& Scheffer, M. (2013). Ideação Suicida, Resolução de Problemas, Expressão de Raiva e Impulsividade em Dependentes de Substâncias Psicoativas.

Tabela 1

Comparação da Expressão de Raiva, Impulsividade e Estratégias de Resolução de Problemas entre os Grupos

\begin{tabular}{|c|c|c|c|}
\hline \multirow[b]{2}{*}{ Variáveis } & \multicolumn{2}{|c|}{ Grupos } & \multirow[b]{2}{*}{$p<$} \\
\hline & $\begin{array}{r}\text { Controle } \\
M(D P)\end{array}$ & $\begin{array}{r}\text { Dependente } \\
M(D P)\end{array}$ & \\
\hline \multicolumn{4}{|l|}{ STAXI } \\
\hline Estado de Raiva & $10,56(0,91)$ & $11,60(3,32)$ & 0,143 \\
\hline Traço de Raiva & $16,08(3,62)$ & $22,56(6,17)$ & $0,001 *$ \\
\hline Temperamento & $6,12(1,92)$ & $9,28(3,07)$ & $0,001 *$ \\
\hline Reação & $7,08(1,97)$ & $9,64(2,94)$ & $0,001 *$ \\
\hline Raiva para Dentro & $15,60(3,59)$ & $17,64(4,34)$ & 0,077 \\
\hline Raiva para Fora & $12,56(3,85)$ & $13,80(4,77)$ & 0,318 \\
\hline Controle de Raiva & $23,52(5,05)$ & $17,44(4,59)$ & $0,001 *$ \\
\hline Expressão de Raiva & $21,96(7,41)$ & $29,68(8,82)$ & $0,002 *$ \\
\hline \multicolumn{4}{|l|}{ BARRAT } \\
\hline Impulsividade Total & $58,96(7,93)$ & $74,84(10,35)$ & $0,001 *$ \\
\hline Atenção & $18,72(2,79)$ & $21,52(3,04)$ & $0,001 *$ \\
\hline Impulsividade Motora & $19,44(4,39)$ & $24,92(5,21)$ & $0,001 *$ \\
\hline Falta de Planejamento & $20,80(3,70)$ & $28,40(5,06)$ & $0,001 *$ \\
\hline \multicolumn{4}{|l|}{ Estratégias de Solução de Problemas } \\
\hline Ensaios Administrados** & $100,56(24,08)$ & $153,64(203,99)$ & 0,208 \\
\hline Número Total Correto & $68,48(13,22)$ & $70,20(15,08)$ & 0,670 \\
\hline Respostas Perseverativas & $14,48(10,14)$ & $15,20(10,99)$ & 0,811 \\
\hline Erros Perseverativos & $12,88(8,80)$ & $11,33(9,41)$ & 0,556 \\
\hline Erros não Perseverativos & $19,32(22,03)$ & $31,66(19,65)$ & $0,044^{*}$ \\
\hline Respostas de Nível Conceitual & $57,68(18,22)$ & $32,29(25,13)$ & $0,001 *$ \\
\hline Número de Categorias Completadas & $4,64(2,21)$ & $3,92(2,21)$ & 0,256 \\
\hline Ensaios p/ Completar Primeira Categoria & $16,52(16,81)$ & $22,68(23,69)$ & 0,295 \\
\hline Fracasso Manter Contexto & $0,76(1,20)$ & $2,33(4,32)$ & 0,097 \\
\hline Aprendendo a Aprender & $3,08(8,03)$ & $0,47(4,94)$ & 0,174 \\
\hline
\end{tabular}

Nota. $M=$ média; $D P=$ desvio padrão.

*Resultado estatisticamente significativo.

** Número total de cartas utilizadas durante a aplicação do teste.

to, um fator relevante é o processo de tratamento no qual o grupo estudado estava submetido no momento da coleta de informações, enquanto os sujeitos dos estudos encontrados, ainda estavam fazendo uso de drogas e não estavam em tratamento.

Num estudo realizado nos EUA com 777 pacientes encaminhados para avaliação de dependência química em um serviço de Emergências Psiquiátricas os resultados mostraram que mais da metade dos pacientes $(55,26 \%)$ apresentaram ideação suicida. Destes, $85 \%$ relataram consumo de cocaína, o que sugere que a ideação suicida pode estar intimamente relacionada ao consumo de drogas, estando à cocaína, mais presente do que outras substâncias de abuso (Garlow, Purselle, \& D'Orio, 2003). No presente estudo, a amostra apresentou elevada frequência de consumo de cocaína e crack, consumindo a droga todos os dias durante alguns anos. A amostra estudada apresentou também associação positiva do tempo de uso do crack com a presença de ideação suicida, confirmando a considerável influência da droga para o surgimento do pensamento suicida, sendo o crack, substância derivada da cocaína. Esse resultado sugere que com o passar dos anos, os dependentes da droga parecem ficar mais propensos a apresentarem ideação suicida assim como, tais pensamentos não tratados podem prolongar ainda mais o tempo de uso e aumentar a gravidade dos episódios depressivos.

No estudo de Garlow et al. (2003) foi verificada a presença de ideação suicida em dependentes químicos como também foi observado que a droga mais utilizada por eles foi a cocaína, resultado que sustenta o percentual encontrado em nossa pesquisa, onde $92 \%$ dos sujeitos entrevistados eram usuários de cocaína. Outro estudo que mostra resultados semelhantes ao estudo em questão é uma pesquisa realizada nos EUA, com 990 consumidores de drogas que verificou que homens mostraram grande probabilidade de apresentar um quadro de Transtorno de Personalidade Antissocial e altas taxas de ideação suicida. Neste estudo, foi observado que homens com ideação suicida foram mais propensos do que ho- 
Tabela 2

Correlações entre Ideação Suicida e Expressão de Raiva, Impulsividade e Estratégias de Solução de Problemas, no Grupo de Dependentes

\begin{tabular}{lcc}
\hline Variáveis & Ideação Suicida $(r)$ & $p<$ \\
\hline Expressão da Raiva & & \\
Estado de Raiva & 0,074 & 0,725 \\
Traço de Raiva & 0,501 & 0,126 \\
Temperamento & 0,314 & $0,008^{*}$ \\
Reação & 0,516 & 0,145 \\
Raiva para Dentro & 0,300 & 0,472 \\
Raiva para Fora & 0,151 & 0,257 \\
Controle de Raiva & $-0,236$ & $0,013^{*}$ \\
Expressão de Raiva & 0,488 & \\
Impulsividade & & 0,231 \\
Atenção & 0,248 & 0,084 \\
Motora & 0,352 & $0,042^{*}$ \\
Falta de Planejamento & 0,410 & $0,015^{*}$ \\
Impulsividade Total & 0,482 & \\
Estratégias de Solução de Problemas & & $0,012^{*}$ \\
Ensaios Administrados & 0,494 & 0,419 \\
Número Total Correto & 0,169 & 0,118 \\
Respostas Perseverativas & 0,328 & 1,000 \\
Erros Perseverativos & 0,000 & 0,224 \\
Erros não Perseverativos & 0,258 & 0,872 \\
Respostas de Nível Conceitual & $-0,035$ & 0,085 \\
Número de Categorias Completadas & $-0,350$ & 0,686 \\
Ensaios para Completar Primeira Categoria & 0,085 & 0,096 \\
Fracasso Manter Contexto & 0,348 & 0,900 \\
Aprendendo a Aprender & 0,026 &
\end{tabular}

Notas. $r=$ correlação.

* Resultado estatisticamente significativo.

mens sem ideação suicida para satisfazer critérios de uso de drogas, com destaque ao uso de cocaína.

Quanto à expressão de raiva, avaliada através do Inventário de Expressão de Raiva como Estado e Traço (STAXI), comparando os dois grupos, os resultados estatisticamente significativos no grupo dos dependentes foram traço de raiva, temperamento, reação e expressão da raiva. Os dependentes de substâncias psicoativas do presente estudo apresentaram uma média de 22,56 pontos para traço de raiva $(D P=6,17)$ o que está acima da média padrão para homens brasileiros, segundo Biaggio (1992/2003), que é de $M=18,92$. Também com média acima do padrão para homens brasileiros, segundo a mesma autora, estão apontando as subescalas, temperamento, com média igual a 9,28 $(D P=2,94)$, sendo o padrão para homens brasileiros $M=6,86$. A reação, com média igual a 9,64 $(D P=2,94)$, quando o padrão é $M=8,94$ e expressão de raiva, com média igual a 29,68 $(D P=8,82)$ quando a média padrão é $M=24,66$.

A partir da escala "Traço de Raiva" foi desenvolvido as subescalas de "Temperamento Raivoso" e "Reação de Raiva", ligadas à maneira de reagir às situações pro- vocadoras ou frustradoras. Dentro dessa perspectiva, pessoas com altos escores em "temperamento raivoso" seriam as chamadas "explosivas", impulsivas, e tenderiam a expressar a raiva com pouca provocação. Já os altos índices em "reação de raiva" estariam ligados a pessoas que tendem a reagir mais violentamente quando criticadas ou provocadas (Biaggio, 1998).

A subescala Controle de Raiva, fator que demonstra a exigência de um investimento de energia no monitoramento e prevenção da experiência e expressão da raiva, sendo desejável para um convívio social mais adaptado (Biaggio, 1992/2003), aparece com baixo resultado, em comparação ao grupo controle, o que expressa o menor grau de controle da raiva que existe nos dependentes entrevistados, corroborando com vários achados que têm indicado que o aumento do comportamento agressivo possui um destacado papel na mediação entre dependência química e ideação suicida (Brent et al., 1996; Dumais et al., 2005; McGirr, Renaud, et al., 2007).

A correlação entre ideação suicida e expressão de raiva no grupo de dependentes teve como resultado significativo, o traço de raiva, a reação e a expressão de raiva. 
Ressalta-se o número significativo de dependentes com ideação suicida que possuem uma maior disposição para perceber várias situações como desagradáveis ou frustradoras, tendendo a reagir sobre elas, do que aqueles dependentes sem ideação suicida, resultados semelhantes são encontrados em outros estudos já realizados (Gil, 2002; Hartwell, 2001; Marzuk, Hortwell, Leon, \& Portera, 2005; Palmini, 2004; Portuguez \& Charchat, 1998).

Em relação à impulsividade, na comparação de médias entre o grupo controle e de dependentes, os resultados significativos foram para as sub-variáveis: atenção, que se caracteriza por pensamentos rápidos e instáveis; atividade motora, que se caracteriza por uma tendência a ações impetuosas; falta de planejamento e orientação para o futuro e impulsividade, todos expressivos no grupo de dependentes. Uma revisão de literatura realizada em 2001, com o objetivo de discutir a relação da impulsividade em transtornos psiquiátricos apontaram que níveis significativamente mais altos de impulsividade são encontrados entre pacientes com transtornos por uso de substâncias, em comparação com outros pacientes, ou pessoas saudáveis (Moeller, Barratt, Dougherty, Schmitz, \& Swann, 2001).

Outros estudos sobre impulsividade em dependentes de substâncias psicoativas, também têm apoiado a relação entre impulsividade e abuso de substâncias. Grande parte destes estudos também utilizou questionários de medidas de impulsividade e encontraram níveis mais altos de impulsividade em dependentes de substâncias psicoativas, em comparação a indivíduos saudáveis. Como também colocam que os dependentes de múltiplas substâncias são mais impulsivos do que aqueles dependentes de uma única substância (Allen, Moeller, Rhoades, \& Cherek, 1998; Moss, Yao, \& Panzak, 1990; O’Boyle \& Barratt, 1993; Patton, Stanford, \& Barratt, 1995). No presente estudo, as drogas mais utilizadas pela grande maioria foram cigarro, cocaína, crack, maconha e álcool o que mostra que a maioria dos dependentes eram poliusuários, corroborando com os dados do estudo acima citado.

Quando foi relacionada a ideação suicida e impulsividade, os resultados mostraram significância estatística positiva para falta de planejamento e impulsividade entre os dependentes com ideação suicida, resultados que podem ser apoiados por alguns estudos, como o apresentado por Kellermann et al. (1992) e Lesage et al. (1994) onde colocam que mais da metade dos casos das pessoas que cometem suicídio preenchem critérios diagnósticos para transtornos de personalidade do grupo B, principalmente, Transtorno da Personalidade Limítrofe e Antissocial, dois quadros caracterizados pela importante presença de traços impulsivos e agressivos. Achados estes, consistentes também com o de Malone et al. (1995), que indica que em várias categorias diagnósticas, existe uma correlação positiva entre a presença de traços impulsivos ou impulsivo-agressivos e a tendência a manifestar o comportamento suicida.

Outro estudo, realizado em 2005, com indivíduos que já haviam tentado suicídio e indivíduos controle, apon- tou que, aqueles com tentativas prévias de suicídio apresentaram resultados mais altos para impulsividade se comparados com aqueles que nunca haviam tentado suicídio. O mesmo estudo apontou também, que os indivíduos que tentaram suicídio uma ou mais vezes durante a vida, além de maior impulsividade, apresentavam história de abuso de álcool, mais frequentemente do que os indivíduos sem tentativas prévias de suicídio (Swann et al., 2005).

Em relação à capacidade para gerar estratégias de solução de problemas, o grupo de dependentes cometeu mais erros não perseverativos e menos respostas de nível conceitual. Entretanto, não houve correlação significativa entre ideação suicida e tal função cognitiva no grupo de dependente, exceto no que diz respeito ao número de ensaios administrados, o qual mostrou-se positivamente relacionado. Esse resultado não é corroborado na literatura, na qual mostra que o pensamento suicida associado à dependência de substâncias psicoativas interfere intensamente no desempenho para resolver problemas (Marzuk et al., 2005), sendo que os processos afetivos e reflexivos envolvidos nas funções do lobo frontal em sobreposição à rigidez cognitiva podem influenciar a expressão recorrente do comportamento suicida (Legris \& van Reekum, 2006).

Este achado indica que a dependência de substâncias psicoativas, independente da presença do pensamento suicida, interferiu no desempenho para resolver problemas no que diz respeito à capacidade para reconhecer $\mathrm{e}$ estabelecer os critérios para a classificação das cartasresposta implícitos na tarefa. Tal dificuldade apresentada causou maior de erros, mas o mesmo não foi encontrado quanto à flexibilidade cognitiva relacionada a condutas perseverativas, sendo que o pensamento suicida prejudica a estratégias eficazes para resolução de problemas, fazendo com que ocorram maiores tentativas para se alcançar o objetivo. Dados do estudo de Burton, Vella, Weller, e Twamley (2011) mostraram que indivíduos com transtornos psiquiátricos e ideação suicida apresentaram pobre funcionamento cognitivo e controle inibitório para resolver problemas.

Pode-se verificar através desta pesquisa que alterações comportamentais e emocionais como a impulsividade aumentada e a acentuada expressão de raiva podem estar intimamente relacionadas com a dependência de substâncias psicoativas, assim como também ao comportamento suicida, na presente amostra. No Brasil as características de dependentes de substâncias psicoativas permanecem as mesmas, quando comparadas com estudos feitos em outros países, o que aponta que as diferenças regionais podem não ter influência sobre as alterações estudadas.

\section{Conclusão}

Concluiu-se que a ideação suicida esteve presente em dependentes de substâncias psicoativas do sexo masculino, podendo o efeito da droga ser um importante desencadeador de alterações emocionais e do comporta- 
mento. Os dependentes de substâncias psicoativas não apresentaram alterações cognitivas significativas, porém apresentaram alterações emocionais no que diz respeito à impulsividade e a expressão de raiva.

A capacidade para gerar estratégias de solução de problemas, neste estudo, não apresentou associação significativa com a ideação suicida, porém acredita-se que esta variável precisa ser mais bem investigada. O tempo de abstinência do grupo de dependentes e o fato de estarem em tratamento, pode ter contribuído para melhor desempenho quanto à flexibilidade do pensamento para resolver problemas, variável que não se mostrou significativamente diferente do grupo controle.

Estes resultados servem como subsídios importantes para um assunto que envolve saúde pública e que, portanto, interessa a toda sociedade. Medidas preventivas do comportamento de busca e do consumo da droga e políticas de educação sobre as consequências do uso, assim como, do trabalho em terapia cognitivo-comportamental, através de entrevistas motivacionais e programas de reabilitação destes indivíduos são necessários na busca da redução e minimização dos danos causados pelas substâncias psicoativas. Tratar os transtornos psiquiátricos pode reduzir significativamente à prevalência de suicídio, pois a morbidade não tratada pode ser importante fator de risco para tal comportamento. Dessa forma, a partir destas constatações, mais investigações podem ser feitas explorando outros aspectos relacionados à dependência de substâncias psicoativas e a presença de ideação suicida nesta população.

A partir disso, percebe-se que é de suma importância a execução de mais pesquisas, com amostras maiores enfocando a dependência de substâncias psicoativas, a ideação suicida e a relação destas com alterações emocionais, compreendendo melhor o perfil desta população. No presente estudo, não foram utilizadas medidas estatísticas que permitissem controlar possíveis viéses de confusão, como por exemplo, presença de transtornos psiquiátricos.

\section{Referências}

Allen, T. J., Moeller, F. G., Rhoades, H. M., \& Cherek, D. R. (1998). Impulsivity and history of drug dependence. Drug and Alcohol Dependence, 50, 137-145.

Almeida, S. A., Guedes, P. M. M., Nogueira, J. A., França U. M., \& Silva, A. C. O. (2009). Investigação de risco para tentativa de suicídio em hospital de João Pessoa- PB. Revista Eletrônica de Enfermagem, 11(2), 383-389.

Amorim, P. (2000). Mini International Neuropsychiatric Interview (MINI): Validação de entrevista breve para diagnóstico de transtornos mentais. Revista Brasileira de Psiquiatria, 22(3), 106-115.

Arango, V., Ernsberger, P., Marzuk, P. M., Chen, J. S., Tierney, H., Stanley, M., et al. (1990). Autoradiographic demonstration of increased serotonin 5-HT2 and beta-adre-nergic receptor binding sites in the brain of suicide victims. Archives of General Psychiatry, 47(11), 1038-1047.

Biaggio, A. (1998). Ansiedade e depressão na concepção de C. D. Spielberger. Revista de Psiquiatria Clínica, 25(6), 291-293.
Biaggio, A. (2003). Manual do Inventário de Expressão de Raiva como Estado e Traço (STAXI). São Paulo, SP: Vetor. (Original work published 1992)

Brent, D. A. (1993). Depression and suicide in children and adolescents. Pediatrics in Review, 14(10), 380-388.

Brent, D. A., Bridge, J., Johnson, B. A., \& Connolly, J. (1996). Suicidal behavior runs in families. A controlled family study of adolescent suicide victims. Archives of General Psychiatry, 53(12), 1145-1152.

Burton, C. Z., Vella, L., Weller, J. A., \& Twamley, E. W. (2011). Differential effects of executive functioning on suicide attempts. Journal Neuropsychiatry and Clinical Neuroscience, 23(2), 173-179.

Corruble, E., Damy, C., \& Guelfi, J. D. (1999). Impulsivity: A relevant dimension in depression regarding suicide attempts? Journal of Affective Disorders, 53(3), 211-215.

Cottler, L. B., Campbell, W., Krishna, V. A., CunninghamWilliams, R. M., \& Abdallah A. B. (2005). Predictors of high rates of suicidal ideation among drug users. Journal of Nervous and Mental Disease, 193(7), 431-437.

Cunha, J. A. (2001). Manual da versão em português das Escalas Beck. São Paulo, SP: Casa do Psicólogo.

Cunha, J. A., Trentini, C. M., Argimon, I. L., Oliveira, M. S., Werlang, B. G., \& Prieb, R. G. (2005). Teste Wisconsin de Classificação de Cartas. São Paulo, SP: Casa do Psicólogo.

Diaconu, G., \& Turecki, G. (2008). Family history of suicidal behavior predicts impulsive-aggressive behavior levels in psychiatric outpatients. Journal of Affective Disorders, 113(12), 172-178.

Diekstra, R. F. W., \& Garnefski, W. (1995). On the nature, magnitude and casuality of suicidal behavior on international perspective. Suicide and Life-Threatening Behavior, 25, 36-57.

Diemen, L. V., Szobot, C. M., Kessler, F., \& Pechansky, F. (2007). Adaptation and construct validation of the Barratt Impulsiveness Scale (BIS 11) to Brazilian Portuguese for use in adolescents. Revista Brasileira de Psiquiatria, 29(2), 153156.

Dumais, A., Lesage, A. D., Alda, M., Rouleau, G., Dumont, M., Chawky, N., et al. (2005). Risk factors for suicide completion in major depression: A case-control study of impulsive and aggressive behaviors in men. American Journal of Psychiatry, 162(11), 2116-2124.

Felthous, A. R. (2011). Suicide behind bars: Trends, inconsistencies, and practical implications. Journal of Forensic Sicence, 56(6), 1541-1555.

Filley, C. M., Price, B. H., Nell, V., Antoinette, T., \& Morgan, A. S. (2001). Toward and Understanding of violence: Neurobehavioral aspects of unwarranted physical aggression- Aspen Neurobehavioral Conference Consensus Statement. Neuropsychiatry, Neuropsychology and Behavior Neurology, 14(1), 1-14.

Fuster, J. M. (2002). Frontal lobe and cognitive development. Journal of Neurocytology, 31, 373-385.

Garlow, S. J., Purselle, D., \& D’Orio, B. (2003). Cocaine use disorders and suicidal ideation. Drug and Alcohol Dependence, 70(1), 101-104.

Gil, R. (2002). Neuropsicologia (2. ed). São Paulo, SP: Santos.

Grant, B. F., \& Hasin, D. S. (1999). Suicidal ideation among the United States drinking population: Results from the National Longitudinal Alcohol Epidemiologic Survey. Journal of Studies on Alcohol and Drugs, 60(3), 422-429.

Hartwell, N. E. (2001). The neuropsychology of suicidal in depressed inpatients. Dissertation Abstracts International: Section B. Sciences and Engineering, 66(11-B), 6136. 
Hawton, K., \& Heeringen, K. (2002). The international handbook of suicide and attempted suicide. Chichester, UK: Wiley.

Hrdina, P. D., Demeter, E., Vu, T. B., Sotonyi, P., \& Palkovits, M. (1993). 5-HT uptake sites and 5-HT2 receptors in brain of antidepressant- Free suicide victims/depressives: Increase in 5-HT2 sites in cortex and amygdala. Brain Research, 614(1-2), 37-44.

Kellermann, A. L., Rivara F. P., Somes, G., Reay, D. T., Francisco, J., \& Banton, J. G. (1992). Suicide in home in relation gun ownership. New England Journal Medicine, 327(7), $467-$ 472.

Knesper, D. J. (2003). Guide to Suicide Assessment. Ann Arbor, MI: University of Michigan.

Lecrubier, Y. (2001). The influence of comorbidity on the prevalence of suicidal behaviour. European Psychiatry, 16(7), 395-399.

Legris, J., \& van Reekum, R. (2006). The neuropsychological correlates of borderline personality disorder and suicidal behavior. Canadian Journal of Psychiatry, 51(3), 131-142.

Lesage, A. D., Boyer, R., Grunberg, F., Vanier, C., Morissette, R., \& Menard-Buteau, C. (1994). Suicide and mental disorders: A case control study of young men. American Journal of Psychiatry, 151(7), 1063-1068.

Malone, K. M., Haas, G. L., Sweeney, J. A., \& Mann, J. J. (1995). Major depression and the risk of attempted suicide. Journal of Affective Disorders, 34(3), 173-185.

Mann, J. J. (1998). The neurobiology of suicide. Nature Medicine, 4, 25-30.

Mann, J. J., Oquendo, M., Underwood, M. D., \& Arango, V. (1999). The neurobiology of suicide risk: A review for the clinican. The Journal of Clinical Psychiatry, 60(Suppl. 2), 7-11.

Mann, J. J., Waternaux, C., Hass, G. L., \& Malone, K. M. (1995). Toward a clinical model of suicidal behavior in psychiatric patients. American Journal of Psychiatry, 156, 181-189.

Marzuk, P. M., Hartwell, N., Leon, A. C., \& Portera, L. (2005). Executive functioning in depressed patients with suicidal ideation. Acta Psychiatrica Scandinavica: Journal information, 112(4), 294-301.

McGirr, A., Paris, J., Lesage, A., Renaud, J., \& Turecki, G. (2007). Risk factors for suicide completion in borderline personality disorder: A case-control study of cluster B comorbidity and impulsive aggression. Journal of Clinical Psychiatry, 68(5), 721-729.

McGirr, A., Renaud, J., Seguin, M., Alda, M., \& Turecki, G. (2008). Course of major depressive disorder and suicide outcome: A psychological autopsy study. Journal of Clinical Psychiatry, 69(6), 966-970.

McGirr, A., Renaud, J., Seguin, M., Alda, M., Benkelfat, C., Lesage, A., et al. (2007). An examination of DSM-IV depressive symptoms and risk for suicide completion in major depressive disorder: A psychological autopsy study. Journal of Affective Disorders, 97(1-3), 203-209.

Meneghel, S. N., Victora, C. C., Faria, N. M. X., Lenine, A. De C., \& Falk, J. W. (2004). Características epidemiológicas do suicídio no Rio Grande do Sul. Revista de Saúde Pública, 38(6), 804-810.

Moeller, F. G., Barratt, E. S., Dougherty, D. M., Schmitz, J. M., \& Swann, A. C. (2001). Psychiatric aspects of impulsivity. American Journal of Psychiatry, 158, 1783-1793.

Moscicki, E. H. (1995). Epidemiology of suicidal behavior. Suicide and Life-Threatening Behavior, 25, 25-35.
Moss, H. B., Yao, J. K., \& Panzak, G. L. (1990). Serotonergic responsivity and behavioraldimensions in antisocial personality disorder with substance abuse. Biological Psychiatry, 28, 325-338.

O’Boyle, M., \& Barratt, E. S. (1993). Impulsivity and DSMIII-R personality disorders. Personality and Individual Differences, 14, 609-611.

Ohmann, S., Schuch, B., Konig, M., Blaas, S., Fliri, C., \& Pppow, C. (2008). Self-injurious behavior in adolescent girls. Association with psychopathology and neuropsychological functions. Psychopathology, 41(4), 226-235.

Pages, K. P., Russo, J. E., Roy-Byrne, P. P., Ries, R. K., \& Cowley, D. S. (1997). Determinants of suicidal ideation: The role of substance use disorders. Journal of Clinical Psychology, 58(11), 510-515.

Palmini, A. (2004). O cérebro e a tomada de decisões. In P. Knapp (Ed.), Teoria cognitivo comportamental na prática psiquiátrica (pp. 71-88). Porto Alegre, RS: Artes médicas.

Patton, J. H., Stanford, M. S., \& Barratt, E. S. (1995). Factor structure of the Barratt Impulsiveness Scale. Journal of Clinical Psychology, 51, 768-774.

Pompili, M., Rihmer, Z., Akiskal, H. S, Innamorati, M., Iliceto, P., Akiskal, K. K., et al. (2008). Temperament and personality dimensions in suicidal and nonsuicidal psychiatric inpatients. Psychopathology, 41(5), 313-321.

Portuguez, M. W., \& Charchat, H. (1998). Avaliação neuropsicológica do lobo frontal. In J. C. Costa (Ed.), Fundamentos neurobiológicos das epilepsias: Aspectos clínicos e cirúrgicos (pp. 957-73). São Paulo, SP: Lemos.

Raust, A., Slama, F. R., Mathieu, F., Roy, I., Chenu, A., Koncke, D., et al. (2007). Prefrontal cortex dysfunction in patients with suicidal behavior. Psychological Medicine, 37, 411-419.

Reyes, J. C., Robles, R. R., Colón, H. M., Negrón, J. L., Matos, T. D., \& Calderón, J. M. (2011). Polydrug use and attempted suicide among Hispanic adolescents in Puerto Rico. Archives of Suicide Research, 15(2), 151-159.

Rogers, J. R. (2001). Theoretical grounding: 'The missing link' in suicide research. Journal of Counseling \& Development, 79(1), 16-29.

Romão, M. R., \& Vieira, L. J. E. S (2004). Tentativas suicidas por envenenamento. Revista Brasileira em Promoção da Saúde, 17(1), 14-20.

Swann, A. C., Dougherty, D. M., Pazzaglia, P. J., Pham, B. S. M., Steinberg, J. L., \& Moeller, F. G. (2005). Increased impulsivity associated with severity of suicide attempt history in patients with bipolar disorder. American Journal of Psychiatry, 162, 1680-1687.

Tiet, Q. Q., Ilgen, M. A., Byrnes, H. F. \& Moos, R. H. (2006). Suicide attempts among substance use disorder patients: An initial step toward a decision tree for suicide managemet. Alcoholism, Clinical and Experimental Research, 30(6), 9981005.

Turecki, G. (1999). O suicídio e sua relação com o comportamento impulsivo-agressivo. Revista Brasileira de Psiquiatria, 21(Suppl. 2), 18-22.

Weir, E. (2001). Suicide: The hidden epidemic. Canadian Medical Association Journal, 165(5), 634-636.

World Health Organization. (2007). Suicide Prevention (SUPRE). Geneve, Switzerland: Author. 